\title{
Gaussian-beam mode analysis of reflection and transmission in multilayer dielectrics
}

\author{
Timothy J. Finn,* Neil A. Trappe, and J. Anthony Murphy \\ Experimental Physics Department, National University of Ireland, Maynooth, Kildare, Ireland \\ *Corresponding author: t.finn@oan.es
}

Received July 23, 2007; accepted October 3, 2007;

posted October 23, 2007 (Doc. ID 85491); published December 7, 2007

\begin{abstract}
The analysis of reflections from thin films or dielectric materials can be approached by a matrix method that treats any thin-layer device as a cascade of sequential, zero-thickness reflecting thin-layer surfaces [J. Opt. Soc. Am. A 2, 1363 (1985)]. Our paper presents an alternative method for predicting the reflection/transmission characteristics of such dielectric films in a Fabry-Perot interferometer configuration based on a Gaussianbeam modal analysis within a scattering-matrix framework [in Proceedings of IEE 7th International Conference on Antennas and Propagation (IEE, 1991), Issue 15, p. 201.] We present and validate a scalar Gaussianbeam modal scattering-matrix approach using long-wavelength examples, where diffraction effects are important to model total transmission and reflection characteristics that also include a waveguide modal description of a corrugated horn. For optical beams the same technique is equally applicable, but diffraction is less severe within this framework. This approach is flexible and has many applications within laser optics and in far-infrared or submillimeter-instrumentation optical analysis, where it is possible to incorporate reflections in both waveguide and free space within the description of a whole system. To conclude and verify the accuracy of the technique, experimental measurements taken at $94 \mathrm{GHz}$ are compared with theoretical predictions for a dielectric cavity of polyethylene sheets between corrugated source and detector antennas. (C) 2007 Optical Society of America

OCIS codes: $310.0310,050.2230,050.1940,230.5750,260.3090,260.3160$.
\end{abstract}

\section{INTRODUCTION}

Gaussian-beam mode analysis (GBMA) has proven to be a powerful tool in the analysis of quasi-optical systems [1] (quasi-optical refers to long-wavelength diffractiondominated propagation). To account for reflected power using GBMA, we use a modal scattering technique based on the mode-matching approach adopted in [2] for waveguide propagation, which effectively conserves power across successive junctions when waveguide radii are cascaded together to represent an antenna, for example. This modal scattering technique has been used to accurately predict the field pattern radiated by antennas in a computationally efficient manner [3]. Although it has been developed for waveguide modes, it can be extended to freespace modes, and the same scattering-matrix formulation is used to track all the power transmitted and reflected in accordance with the conservation of power. Reflected and transmitted components can be evaluated from a large variety of optical elements including dielectric sheets, lenses, and mirrors (both planar and curved) as well as truncating apertures, which may or may not be perfectly absorbing. Propagation through free space (or waveguides) is represented by diagonal matrices, where the appropriate phase slippage is introduced for each mode. In an optical system, potential reflecting surfaces are obvious when one is dealing with typical geometrical components. At longer wavelengths we must also consider the feed antenna itself as a potential source of reflection. This complicates analysis, as reflections can occur within the entire length of the antenna itself and not just at the aperture. Fortunately, by extending the full electromag- netic waveguide description of the horn to free space [1], we account for all these potential reflections within a complete optical system. This interconnectivity (waveguide modes to free-space modes) distinguishes this modal approach from other techniques, where the reflected power is modeled as occurring solely between the reflecting surface and the input-horn aperture, neglecting the reflections within the body of the antenna. This technique is discussed in detail in $[1,4]$.

The most common technique for predicting standingwave patterns is based on summation of multiple beams with appropriate phase terms between thin layers $[5,6]$. In [4] it has been demonstrated that the presence of a horn antenna leads to high-Q reflection effects superimposed on the typical sinusoidal ripple associated with standing waves between planar elements. This paper presents a range of dielectric thin-layer configurations illuminated by a fundamental-mode Gaussian and full description of the electromagnetic horn antenna, in comparison to the thin-layer method utilized in [5]. In addition, a planar dielectric Fabry-Perot interferometer is illuminated by a corrugated horn at $94 \mathrm{GHz}$, and experimental measurements are presented to validate the technique when waveguide modes are also included to represent the corrugated horn structure in conjunction with free-space modes.

\section{THEORY}

The scattering-matrix technique can be considered a method of keeping track of the transmitted and reflected 
components by treating an arbitrary optical system as a series of successive subsections, which when added together in their entirety describe propagation within the whole system. Each section is treated as a separate, independent component that is then cascaded with the adjacent subsequent section ultimately to represent the system as a whole [2], as illustrated in Fig. 1.

In the modal description of free space, the modes can be represented by associated Laguerre-Gaussian polynomials when a high degree of cylindrical symmetry is present. Otherwise in the case of asymmetric or Cartesian systems, Hermite-Gaussian polynomials are the preferred choice of mode set. The higher-order Gaussian-beam solutions are given in [7] as

$$
\begin{aligned}
\Psi_{m n}(x, y)= & h_{m}\left(\frac{\sqrt{2} x}{W}\right) h_{n}\left(\frac{\sqrt{2} y}{W}\right) \\
& \times \exp \left[-j k\left(z+\frac{r^{2}}{2 R}\right)\right] \exp \left(j \phi_{m n}\right),
\end{aligned}
$$

with $h_{m}$ the normalized Hermite function of the form

$$
\begin{aligned}
& h_{m}\left(\frac{\sqrt{2} x}{W}\right)=\frac{1}{\sqrt{2^{m-1 / 2}} m ! \sqrt{\pi W^{2}}} H_{m}\left(\sqrt{2} \frac{x}{W}\right) \exp \left(-\frac{x^{2}}{W^{2}}\right), \\
& h_{n}\left(\frac{\sqrt{2} y}{W}\right)=\frac{1}{\sqrt{2^{n-1 / 2}} n ! \sqrt{\pi W^{2}}} H_{n}\left(\sqrt{2} \frac{y}{W}\right) \exp \left(-\frac{y^{2}}{W^{2}}\right),
\end{aligned}
$$

where $W$ is the beam width, $R$ is the phase radius of curvature, and $\phi$ is the phase slippage of each mode. The appropriate normalizing factors are assumed. The electric field can be calculated using a modal description at any plane by a linear combination of the mode coefficients (which determine the amount of power contained in each mode) and equivalent modes (i.e., $\Sigma_{m} A_{m} \Psi_{m}$ ) that are fully determined by the beam width, the phase radius of curvature, and the relative phase slippage between modes. The electric field must be known at an initial plane. Only the phase slippage is mode dependent. The classical $A B C D$ matrix method altered for GBM propagation is used throughout this paper to track the evolution of the GBM parameters through free space [8]. We utilize this technique to track beam evolution in both directions with a scattering-matrix framework, and this is how multiple reflections can be accounted for within an optical system.

Each potentially reflecting optical component is represented by a scattering matrix where $[A]$ and $[B]$ are col-

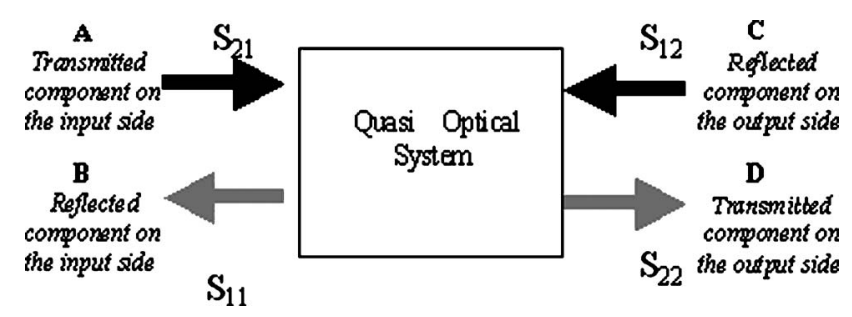

Fig. 1. Optical system represented by the mode-matching concept. umn matrices for the forward-propagating and reflected components as viewed from the input side. $[D]$ and $[C]$ represent the forward and reflected components from the output side. These matrices take the form of rectangular matrices [2], which are related by

$$
\left[\begin{array}{c}
{[B]} \\
{[D]}
\end{array}\right]=\left[\begin{array}{ll}
{\left[S_{11}\right]} & {\left[S_{12}\right]} \\
{\left[S_{21}\right]} & {\left[S_{22}\right]}
\end{array}\right]\left[\begin{array}{l}
{[A]} \\
{[C]}
\end{array}\right]
$$

where $[A]$ and $[B]$ are vectors of mode coefficients containing the transmitted and reflected components on the source side and $[C]$ and $[D]$ are the transmitted and reflected components on the output side. For many systems there will only be one mode at the input (e.g., the $\mathrm{TE}_{11}$ for circular waveguide and the $\mathrm{TE}_{10}$ for rectangular waveguides), so $[A]$ will take the form of a column vector with one component. In any case, the cascade for any two sections is given by

$$
\left[S^{a}\right]=\left[\begin{array}{ll}
{\left[S_{11}^{a}\right]} & {\left[S_{12}^{a}\right]} \\
{\left[S_{21}^{a}\right]} & {\left[S_{22}^{a}\right]}
\end{array}\right], \quad\left[S^{b}\right]=\left[\begin{array}{ll}
{\left[S_{11}^{b}\right]} & {\left[S_{12}^{b}\right]} \\
{\left[S_{21}^{b}\right]} & {\left[S_{22}^{b}\right]}
\end{array}\right],
$$

where $\left[S^{a}\right]$ and $\left[S^{b}\right]$ are the scattering matrices for the two arbitrary sections, with their submatrices representing the reflected and transmitted components of the fields. The cascaded matrix takes the form

$$
\left[S^{c}\right]=\left[\begin{array}{ll}
S_{11}^{c} & S_{12}^{c} \\
S_{21}^{c} & S_{22}^{c}
\end{array}\right] .
$$

The submatrices for $\left[S^{c}\right]$ are calculated by

$$
\begin{gathered}
{\left[S_{11}^{c}\right]=\left[S_{12}^{a}\right]\left[[I]-\left[S_{11}^{b}\right]\left[S_{22}^{a}\right]\right]^{-1}\left[S_{11}^{b}\right]\left[S_{21}^{a}\right]+\left[S_{11}^{a}\right],} \\
{\left[S_{12}^{c}\right]=\left[S_{12}^{a}\right]\left[[I]-\left[S_{11}^{b}\right]\left[S_{22}^{a}\right]\right]^{-1}\left[S_{12}^{b}\right]} \\
{\left[S_{21}^{c}\right]=\left[S_{21}^{b}\right]\left[[I]-\left[S_{22}^{a}\right]\left[S_{11}^{b}\right]\right]^{-1}\left[S_{21}^{a}\right]} \\
{\left[S_{22}^{c}\right]=\left[S_{21}^{b}\right]\left[[I]-\left[S_{22}^{a}\right]\left[S_{11}^{b}\right]\right]^{-1}\left[S_{22}^{a}\right]\left[S_{12}^{b}\right]+\left[S_{22}^{b}\right],}
\end{gathered}
$$

where $[I]$ is the identity matrix. Thus, in order to account for reflections and transmissions within an optical system, we must represent each component or subsection by its equivalent scattering matrix. In this way, the output of each section then becomes the input for the next. If, for example, we wish to analyze the total reflected component from a convex lens, we can represent it as a curved front surface affecting the input beam phase front, followed by a propagation through a medium of refractive index $n$ and finally by a conjugated curved surface. It is also possible to include antireflection coatings by simply including an extra curved surface and equivalent propagation section. Figure 2 illustrates this cascading process.

\section{A. Modal Propagation}

In quasi-optical systems the evolution of the free-space beam can be considered in a number of ways. Most commonly the beam evolves freely via free-space propagation, where the beam is considered to be converging or diverging depending on the phase radius of curvature $(R>0$ signifies divergence and $R<0$ convergence). The propagation 


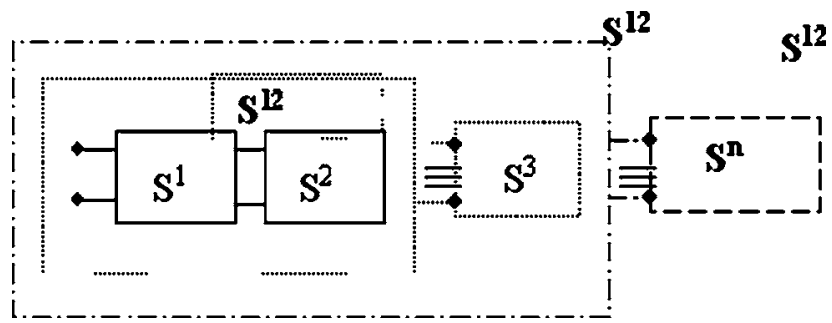

Fig. 2. Cascade of $N$ submatrices yields a full $4 \times 4$ matrix that represents the optical system.

will be represented by a diagonal matrix, which consists of the phase terms in Eq. (1). Each diagonal element will be of the form

$$
V_{m n}=\exp \left[-i k z+i(m+n+1) \Delta \phi_{m n}\right],
$$

where the $k z$ term represents the plane-wave phase delay and the second term $\Delta \phi_{n}$ is the relative phase slippage of the $n$th mode worked out using the $A B C D$ matrix method, i.e., the common on-axis phase difference between successive modes. The propagation scatter matrix is then of the form

$$
\left|\begin{array}{ll}
S_{11} & S_{12} \\
S_{21} & S_{22}
\end{array}\right|_{\text {prop }}=\left|\begin{array}{ll}
0 & V \\
V & 0
\end{array}\right| .
$$

The $S_{11}$ and $S_{22}$ matrices contain only zero elements since a pure propagation step involves no power scattering between modes. When dealing with propagation within a dielectric medium of refractive index $n$, we utilize the reduced radius of curvature convention introduced in [6], illustrated in [1], and discussed in Subsection 2.B.

\section{B. Partially Reflecting/Transmitting Dielectric Sheets}

For a dielectric sheet we consider reflection from the surface, as illustrated in Fig. 3. Upon reflection, the phase front undergoes a radius-of-curvature transformation based on the curvature of the sheet; it will appear to be emanating from a waist located on the output side of the mirror [9]. The beam radius itself is unchanged upon reflection but will now evolve according to the new radius of curvature imposed by the mirror.

The scattering matrix, distributing power between adjacent modes by taking account of the change in curvature upon reflection $S_{m n}$, can then be written as an $m \times n$ matrix and is given by the overlap integral between the input and output fields over the area of the sheet [10],

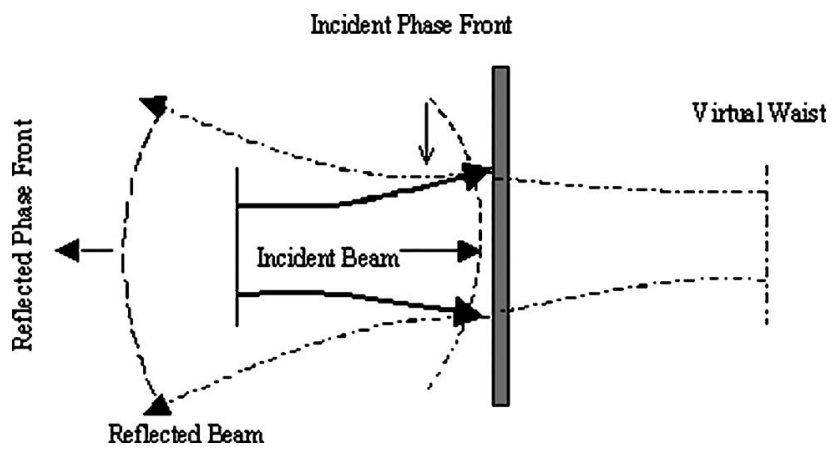

Fig. 3. Reflection of a quasi-optical beam from a dielectric sheet.

$$
\begin{aligned}
S_{m n}= & \int_{A}\left(\psi_{m} \exp \left[\frac{j k r^{2}}{2}\left(\frac{1}{R_{\text {inc }}}-\frac{2}{R_{\text {curv }}}\right)\right]\right)^{*} \psi_{n} \\
& \times \exp \left[-\frac{j k r^{2}}{2}\left(\frac{1}{R_{\text {inc }}}\right)\right] \mathrm{d} x \mathrm{~d} y,
\end{aligned}
$$

where $R_{i n c}$ is the radius of curvature of the incident beam and $R_{\text {curv }}$ the curvature of the dielectric surface. To account for the different refractive indices, we use a concept from geometrical $A B C D$ ray matrices, the reduced radius of curvature, $\hat{R}$ defined in [8] as

$$
\hat{R}=R / n,
$$

where $n$ is the refractive index of the medium and $R$ is the free space radius of curvature at that plane [8]. For geometric optics we can write

$$
\hat{R}_{2}=\frac{A \hat{R}_{1}+B}{C \hat{R}_{1}+D},
$$

where $\hat{R}_{1}$ is the radius of curvature in medium one and $\hat{R}_{2}$ is the radius of curvature in medium two, as illustrated in Fig. 4. This equation allows us to calculate the change in radius of curvature due to a transformation by a system described by an $A B C D$ ray matrix. This formula is valid only for geometrical optics. In order to convert this spherical point-source wave to represent the Gaussian beam, we must use the complex reduced beam parameter, which accounts for the finite extent of the source but is generalized for a medium of refractive index $n$ [8]. We find this to be

$$
\frac{1}{\hat{q}} \equiv \frac{n}{q} \equiv \frac{n}{R(z)}-j \frac{n \lambda}{\pi W(z)^{2}}=\frac{1}{\hat{R}}-j \frac{\lambda_{0}}{\pi W(z)^{2}},
$$

where $R(z)$ is the free-space radius of curvature, $W(z)$ is the beam radius, and $\lambda$ is given by $\lambda_{0} / n$, where $n$ is the local refractive index of the media and $\lambda_{0}$ is the wavelength in free space. The paraxial-wave transformation rule using the $A B C D$ matrix elements and the $\hat{q}$ Gaussian-beam parameter then becomes

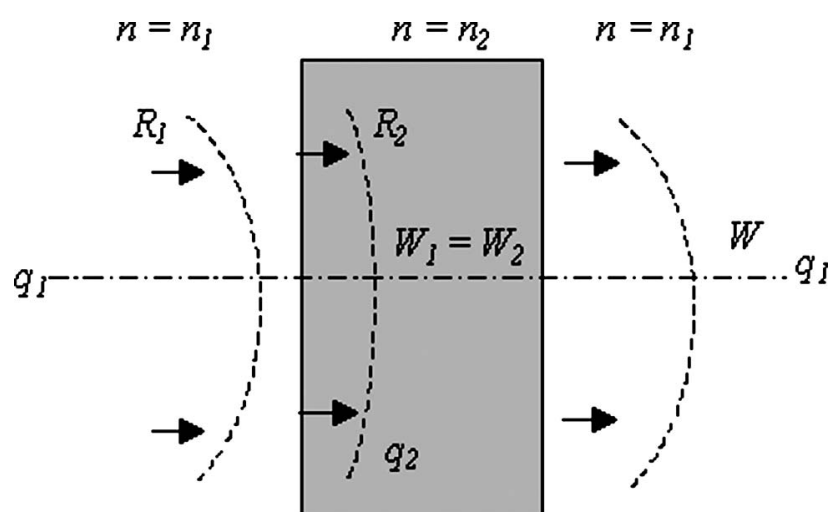

Fig. 4. Effect on the Gaussian-beam parameters of propagation through a dielectric interface. 


$$
\hat{q}_{2}=\frac{A \hat{q}_{1}+B}{C \hat{q}_{1}+D}
$$

where the subscript on $q$ values refers to the medium in which the wave is traveling.

By using the reduced $q$ value we can carry out all beam transformation calculations using solely the local index of refraction $n$ and $\lambda_{0}$. The radius of curvature will scale by a factor of $1 / n$, but the beam radius remains unchanged in the new medium. We can alter two of the matrices described in [11] to describe propagation in a dielectric medium. First, the propagation matrix must be altered to account for the refractive index and is now given as $\left\{1, L n_{1} / n_{2}, 0,1\right\}$ where $L$ is the distance traveled, $n_{1}$ is the index of refraction of the first medium, and $n_{2}$ is the index of refraction of the second medium. The curved dielectric interface of normal incidence is given as $\left\{1,0,\left(n_{2}\right.\right.$ $\left.\left.-n_{1}\right) / R, 1\right\}$. Cascading these components will give a full $A B C D$ description of the system, including the effects of the change in refractive index. The scattering matrix is then given by

$$
\left[\begin{array}{ll}
r_{i j} S_{11} & S_{12} t_{i j} \\
t_{i j} S_{21} & S_{22} r_{i j}
\end{array}\right]
$$

where $r_{i j}$ and $t_{i j}$ are the reflection and transmission coefficients and are calculated via the Fresnel formulas for normal incidence as given by [6]

$$
r_{i j}=\frac{n_{i}-n_{j}}{n_{i}+n_{j}}, \quad t_{i j}=\frac{2 n_{i}}{n_{i}+n_{j}},
$$

where the reflectivity $R=\left|r_{i j}\right|^{2}$ and the transmittivity $T$ $=\left|t_{i j}\right|^{2}$ and $i, j$ represent the media $i$ and $j$ of refractive index $n_{i}$ and $n_{j}$ (e.g., $r_{12}$ represents the reflection from the interface between mediums 1 and 2 while $t_{23}$ represents the transmission at the interface between mediums 2 and 3 ) with the usual relationship of $R+T=1$. In addition, there will be propagation through the medium that constitutes the dielectric medium that may be handled by the propagation matrix of Subsection 2.A. The finite size of optical components can be determined by the limit of the overlap integral between the field incident and the output field. All radiation within the aperture $\left[\left(x^{2}+y^{2}\right)<a\right]$ is included, and all radiation outside the aperture limits $\left[\left(x^{2}\right.\right.$ $\left.\left.+y^{2}\right)>a\right]$ can be considered lost. The finite size of mirrors can be handled in a similar manner, where all power incident on the surface is reflected and all power outside the mirror rim is truncated.

\section{Modeling Horn Antennas and Free-Space \\ Transformation}

A software program called sCATTER [3] has been developed that allows the prediction of the full EM reflection/ transmission properties of homogeneous and inhomogeneous horn antennas with cylindrical geometry as are frequently used in $\mathrm{GHz} / \mathrm{THz}$ systems. This describes the radiated fields in terms of TE and TM modes. In order to extend the waveguide-mode description of the antenna to the free-space mode equivalent, a transformation matrix is used from $[1,4]$ that allows the interconnectivity of the two mode sets within a scatter-matrix framework. This is achieved via a free-space transformation matrix that scatters power from the TE/TM mode set to a HermiteGaussian mode set [1]. Utilizing the output of SCATTER describing transmission/reflection from a particular horn geometry, the transformation matrix and the matrices of Subsections 2.A and 2.B result in the analytical tools necessary to represent many typical submillimeter and far inrared components.

\section{DUAL THIN-LAYER REFLECTIONS}

If we consider the propagation of an EM wave through a homogeneous thin film as shown in Fig. 5, we find from [6] that the total reflection $R$ and transmission $T$ of a dual-layer sheet is given by

$$
\begin{aligned}
R & =|r|^{2}=\frac{r_{i j}^{2}+r_{j k}^{2}+2 r_{i j} r_{j k} \cos (2 \beta)}{1+r_{i j}^{2} r_{j k}^{2}+2 r_{i j} r_{j k} \cos (2 \beta)} T=|t|^{2} \\
& =\frac{n_{3} \cos \left(\theta_{3}\right)}{n_{3} \cos \left(\theta_{1}\right)} \frac{t_{i j}^{2} t_{j k}^{2}}{1+r_{i j}^{2} r_{j k}^{2}+2 r_{i j} r_{j k} \cos (2 \beta)},
\end{aligned}
$$

where $\beta=2 \pi n_{\text {med }} h \cos \left[\theta_{\text {med }}\right] / \lambda . i, j, k$ refer to the medium in which the radiation is currently propagating, labeled 1 , 2, and 3 in Fig. 5. This situation is relevant to lenses and cryostat windows in quasi-optical systems where $n_{1}=n_{3}$ $=1$ and $n_{2}$ will depend on the material used.

If we replace $\beta$ with $\beta+\pi$, there is no alteration in $R$ or $T$. Furthermore, if we replace $h$ with $h+\Delta h$, where

$$
\Delta h=\frac{\lambda_{0}}{2 n_{2} \cos \theta_{2}},
$$

the reflectivity and transmittivity remain unchanged when two films differ in thickness by an integral multiple of $\lambda_{0} / 2 n_{2} \cos \theta_{2}$. It can be shown that when $n_{2} h$ $=m \lambda_{0} / 4 \cos \theta_{2}$, the reflection is at a minimum when $m$ is odd and a maximum when $m$ is even, depending on the relative values of $n_{1}, n_{2}$, and $n_{3}$ :

$$
\text { If }(-1)^{m}\left(n_{1}-n_{2}\right)\left(n_{2}-n_{3}\right)>0 \text {, we find a maximum. }
$$

$$
\text { If }(-1)^{m}\left(n_{1}-n_{2}\right)\left(n_{2}-n_{3}\right)<0 \text {, we find a minimum. }
$$

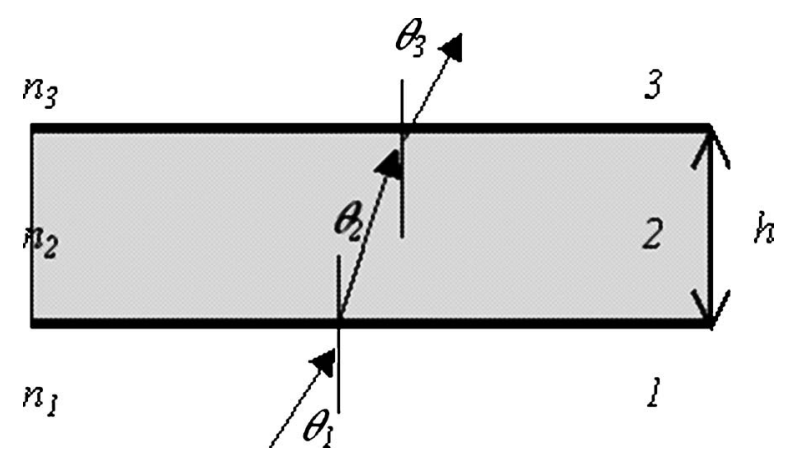

Fig. 5. Propagation of an EM wave through a homogenous film. The arrows represent light rays propagating through the respective media. 
The above conditions can be applied to many classical dielectric configurations. Two specific examples were chosen to highlight the ability of Gaussian-beam mode scattering theory to replicate the results of Eq. (19).

\section{A. Reflectivity as a Function of Path Length}

First, a configuration was chosen, where

- a dielectric with a refractive index $n_{2}=3$ was selected with $n_{1}=n_{3}=1$ (corresponds to sapphire layer of material in air);

- the wavelength was set to $\lambda=500 \mu \mathrm{m}$ with an input Gaussian beam profile of waist $W=1 \mathrm{~mm}$ located at the first sheet and at normal incidence to the sheet.

Sapphire is a material often used in submillimeter systems. Recalling Eq. (19), since $n_{2}>n_{3}$, we find a maximum located at odd integer multiples of $\lambda / 4$. The scattering-matrix model was constructed using the matrices described in Subsections 2.A-2.C using the following steps:

1. Propagation of the beam in free space to the dielectric sheet interface. This is a fundamental Gaussian input.

2. Accounting for the dielectric sheet reflectivity (i.e., the curvature of the surface, although in this example $R_{\text {dielec }}=\infty$, and a truncation of diameter $6 \mathrm{~W}$ due to the finite size of the sheet).

3. Propagation within the medium and potential reflections from the back face of the dielectric.

Figure 6 shows the classical theory and the scatteringmatrix equivalent, which exhibit excellent agreement, validating the accuracy of the scattering-matrix method.

The second configuration was as follows:

A dielectric with a refractive index $n_{2}=1.1$ (corresponds to Styrofoam) and $n_{3}=1.5$ (corresponds to HDPE) was then selected.

The wavelength was set to $\lambda=500 \mu \mathrm{m}$ with an input waist $W=1 \mathrm{~mm}$ at normal incidence to the sheet and again located at the sheet surface.

High-density polyethylene (HDPE) and Styrofoam are again typical submillimeter materials. Again, recalling from Eq. (19), since $n_{2}<n_{3}$ we find a maximum located at even integer multiples of $\lambda / 4$. Figure 7 displays the classical theory and the scattering-matrix equivalent, which

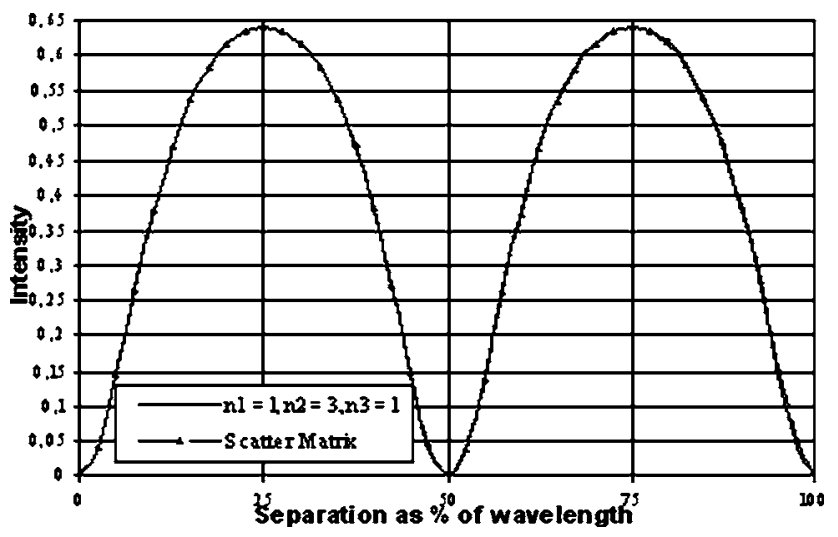

Fig. 6. Reflectivity of a dielectric film of refractive index $n$ as a function of optical thickness for the first configuration (see text).

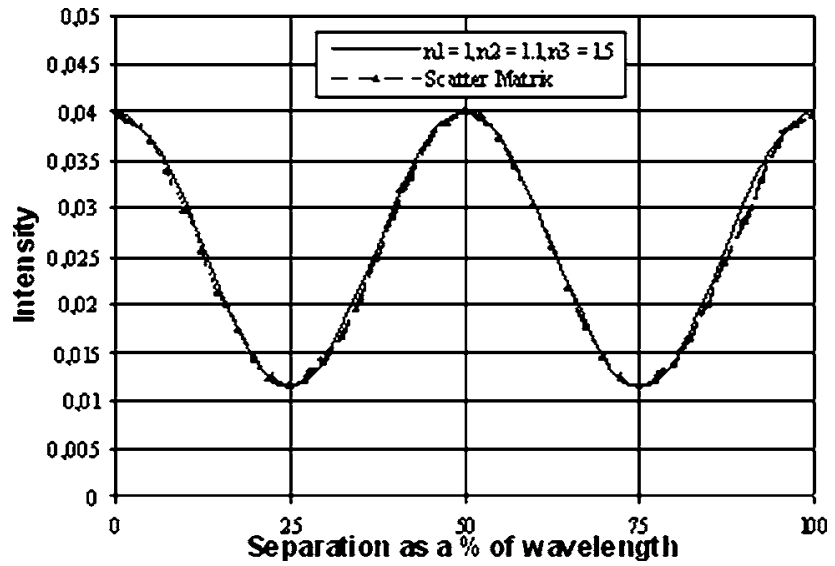

Fig. 7. Reflectivity of a dielectric film of refractive index $n$ as a function of optical thickness for the second configuration (see text).

again shows excellent agreement while recreating the switch between odd and even integer multiples in Eq. (19), further validating the accuracy of the modal description.

\section{B. Reflectivity as a Function of Refractive Index}

For lenses in general, $n_{1}=n_{3}$ and the medium has a refractive index of $n_{2}$, which alters the reflectivity of the sheet. For a Fabry-Perot interferometer (see Section 4) the condition $n_{1}=n_{3}$ is often found, although $n_{3}$ can also vary depending on which substance is present. The sheet has a fixed thickness of $\lambda / 4$ and the same initial parameters as for the configurations described above except that now the refractive index, rather than thickness, is varied. The reflectivity calculated via Eqs. (16) and (17) is illustrated in Fig. 8 along with the scattering-matrix equivalent, which shows excellent agreement with the classical theory (the top trace corresponds to $n_{1}=1, n_{3}=1$, the second trace to $n_{1}=1, n_{3}=1.45$, etc.).

The reflectivity of the sheet is expected to be zero when $n_{2}=\sqrt{n_{1} n_{3}}$, and Fig. 6 demonstrates this particular characteristic where the reflectivity goes to zero for a particular value of $n_{2}$ depending on the refractive index of $n_{1}$ and $n_{3}$ (e.g., for $n_{1}=n_{3}=1$, then $n_{2}=1.22$ ). Thus by coating surfaces with refractive indices of high refractive index, we can obtain very high reflection coefficients, or alterna-

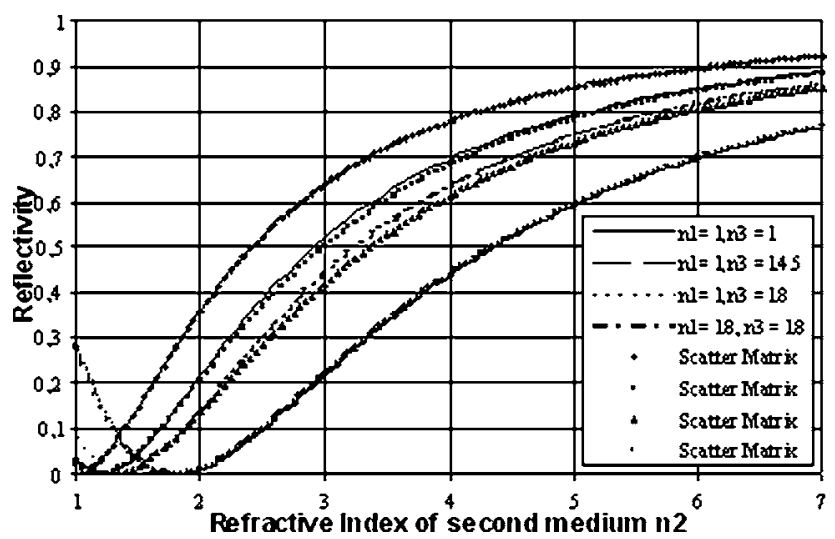

Fig. 8. Reflectivity response of a quarter-wavelength-thick dielectric as a function of the refractive index of the second medium. 
tively we can make antireflection coatings by choosing a quarter-wavelength thickness and by choosing a refractive index $n_{2}$ that is lower than that of the refractive index of the last medium $n_{3}$ (the lens) and where $n_{1}=1$ (air).

The reflectivity, and hence the transmission of a dielectric sheet, is highly dependent not only on the refractive index but also on the thickness of the dielectric sheet. This relationship is further complicated by the relative refractive indices of the medium surrounding the lens.

\section{FABRY-PEROT INTERFEROMETER}

Fabry-Perot interferometers (FPIs) find multiple uses in submillimeter instrumentation, particularly as diplexers or frequency-selective filters [11]. They consist of two semireflecting surfaces separated by a distance $d$, as illustrated in Fig. 9. The FPI passes and partially reflects multiple beams, and summations of these beams interfere constructively or destructively for various path lengths, thus determining the overall transmission characteristics of the device.

Due to their potential high resolution, frequencyselective FPIs are often used as filters, which have the advantage of variable-frequency bandpass and whose resonance characteristics can be altered by varying the refractive index of the FPI. Multiple FPIs may be used to realize a broader spectral range and increased resolution and to achieve a ripple-free transmission profile. They are also used in diplexer arrangements, as they may also be used for superimposing two signals in a heterodyne instrument, but this requires two separate FPIs to avoid losing one polarization [11]. A FPI can also be used as a polarization rotator by the use of two quarter-wave plates, which transform a linear polarization to a circular polarization of a certain handedness.

The finesse of the FPI, which determines the spectral resolution of the device or, alternatively, the ratio of fringe spacing to the FWHM, is given by [11]

$$
F=\frac{\pi\left|r^{2}\right|}{1-\left|r^{2}\right|} \text {. }
$$

To validate the scattering-matrix theory, an alternative method for calculating the behavior of the FPI presented in [1] is utilized. Briefly, this theory is based on the analysis of multilayer films ignoring absorptive, scattering, misalignment and diffraction losses and with a normal

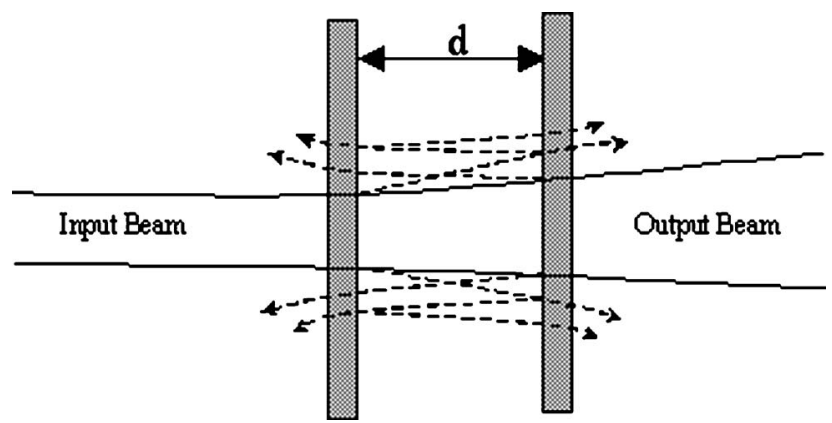

Fig. 9. Fabry-Perot interferometer with an incident Gaussian beam. The effect of the multiple reflections is to confine a longer free-space propagation to a smaller distance. angle of incidence. In addition, the thickness of the sheets is considered to be zero with just the reflectivity and the distance between sheets as variables. If we consider Fig. 10 , where we have $N$ partially transmitting sheets going from $i=1$ to $i=N$, we find that the amplitude of the electric field at the left side of mirror 1 and propagating to the right is $E_{i}^{+}$and that the reflected component of the field on the same side and propagating to the left is $E_{i}^{-}$. We also find that the reflection and transmission coefficients are $r_{i}$ and $t_{i}$, respectively, and the distance from sheet $i$ to sheet $i+1$ is $L_{i}$. The phase length from sheet $i$ to $i+1$ is given by $\beta_{i}=2 \pi L_{i} / \lambda$. The transmission coefficient $t$ for a stack of $N$ mirrors is given by

$$
t=\frac{E_{N+1}^{+}}{E_{1}^{+}}=\frac{t_{N}}{E_{N}^{+}}=\frac{t_{1} t_{2} \ldots t_{N} E_{N}^{+}}{A E_{N}^{+}+B E_{N}^{-}}=\frac{t_{1} t_{2} \ldots t_{N}}{A+r_{N} B},
$$

where $t_{1,2 \ldots N}$ represents the transmission coefficient of sheet $1,2 \ldots N$, and $A$ and $B$ are related to the phase length from sheet to sheet.

\section{A. Two-Mirror Fabry-Perot Interferometer} For the two-mirror FPI we obtain the result

$$
T=|t|^{2}=\frac{t_{1}^{2} t_{2}^{2}}{1+r_{1}^{2} r_{2}^{2}+2 r_{1} r_{2} \cos \left(2 \beta_{1}\right)},
$$

where $T$ is the total transmittivity of the sheet.

In the scattering-matrix approach we treat the FPI as two dielectric slabs of refractive index $n$, of finite thickness, and with a layer of air sandwiched between them. Therefore the model will be more representative since the FPI consists of two dielectric sheets of finite thickness as shown in Fig. 9, whereas the multilayer approach essentially ignores this and treats them as infinitely thin sheets, as shown in Fig. 8. Thus we will have seven subsections:

$$
\begin{aligned}
\text { Sheet Interface } & \rightarrow \text { Medium } \rightarrow \text { Sheet Interface } \rightarrow \text { Air } \\
& \rightarrow \text { Sheet Interface } \rightarrow \text { Medium } \\
& \rightarrow \text { Sheet Interface. }
\end{aligned}
$$

The initial matrix in the cascade is calculated via Eq. (10) with the reflection and transmission coefficients calculated by Eq. (16). Propagation through the medium of

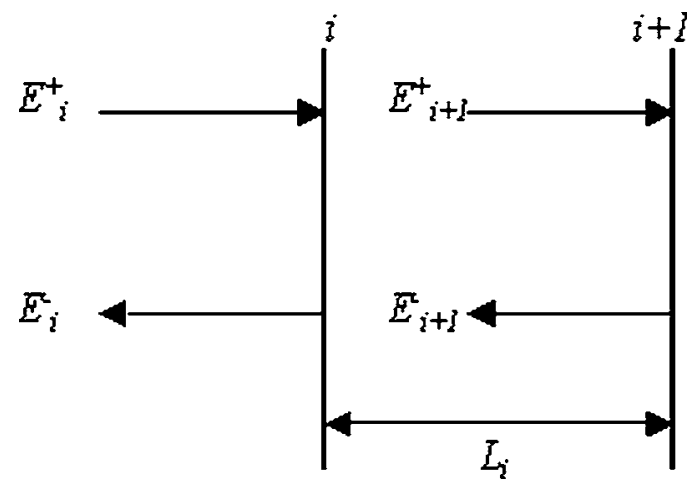

Fig. 10. Amplitudes at partially transmitting sheets $i$ and $i+1$ for a succession of $N$ sheets. 
index $n$ can be handled by the propagation matrix of Subsection 2.A using a diagonal matrix, accounting for the index of refraction of the medium. The $A B C D$ matrix method is used to track the evolution of the GBM parameters $W, R$, and $\phi$. The transmitted power corresponds to the $S_{21}$ matrix and the reflected power to the $S_{11}$ matrix (see Fig. 1).

Figure 11(a) shows the frequency response of the FPI as the distance between the sheets is increased for a fundamental Gaussian waist input located at the surface of the first sheet and with zero distance between the front and back face of either sheet. The dielectric sheet thickness is $\lambda / 4$, and the input beam has a beam width $W$ $=5 \mathrm{~mm}$ and a wavelength of $\lambda=3 \mathrm{~mm}$. All the dielectric sheets are $4 W$ or greater in diameter.

The refractive index of the second medium was set to 5.62 , yielding $R=0.88$ and $T=0.12$. This is the index of refraction of thallium bromide-iodide (KRS-5) at $100 \mathrm{GHz}$, yielding a finesse of 13.4. Figure 11(b) illustrates a second example for values of $R=0.1$ and $T=0.9$, with the refractive index of the second medium equal to 1.39 , which is the index of refraction of Teflon, yielding a finesse of 0.032. Both KRS-5 and particularly Teflon are dielectric substances that are frequently used in submillimeter optical systems as filters and lenses and cryostat windows. The FPI exhibits the classical $\lambda / 2$ dependence for transmission as predicted by Eq. (19), with the reflection char-

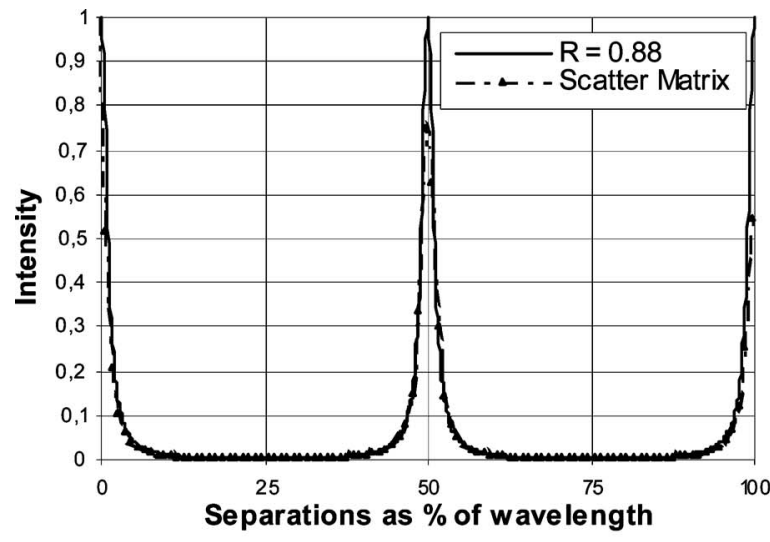

(a)

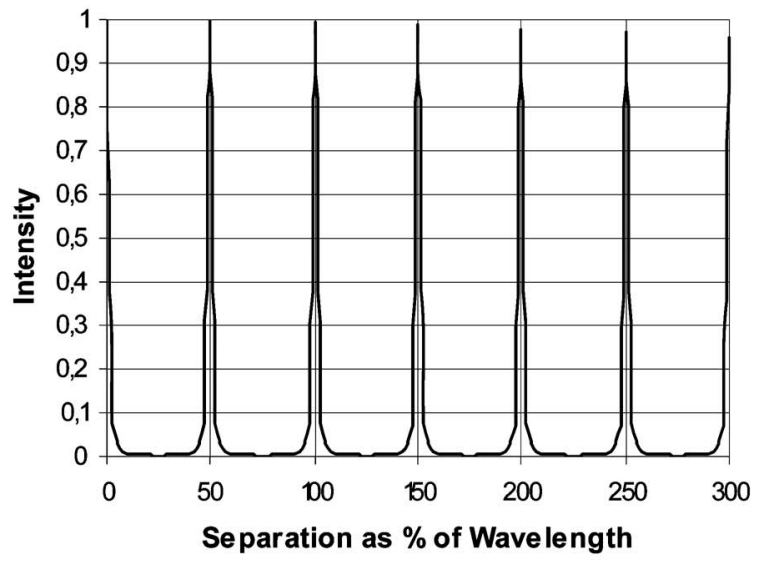

(c) acteristic as the reciprocal of $T$ with a $\lambda / 4$ dependence. It also exhibits the diffractive losses due to the longwavelength nature of the radiation, which can be seen as a decrease in peak intensity as the separation increases. Also shown for comparison in Fig. 11 is the evaluation of Eq. (22). Excellent agreement is found between the two approaches except that the multilayer approach neglects diffractive losses. This is one of the major benefits of adopting the GBM approach, which is parameterized by an evolving beam width, radius of curvature, and phase term. The plane-wave approach in [5], by contrast, simply considers the phase evolution from component to component, which is appropriate in the geometrical limit $(\lambda$ $\rightarrow 0$ ) but gives rise to discrepancies at longer wavelengths. However, Fig. 11(c) illustrates that as the wavelength is reduced to $\lambda=30 \mu \mathrm{m}$ in the scattering-matrix technique, the diffractive losses become small in comparison with Fig. 11(d), which illustrates the diffractive losses that occur as the range is extended over several wavelengths for $\lambda=3 \mathrm{~mm}$. This is the behavior that one would expect for a quasi-collimated beam at $30 \mu \mathrm{m}$. The reflectivity $R=0.9$ for Figs. 11(c) and 11(d).

\section{B. Three-Mirror Fabry-Perot Interferometer}

In general, while FPIs are highly useful as narrow passband filters, one would like to be able to alter the free spectral range and obtain higher resolution. In this re-

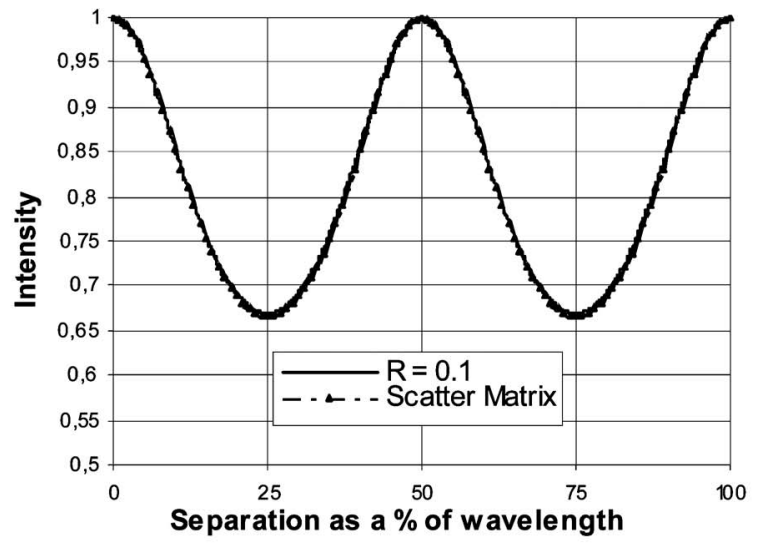

(b)

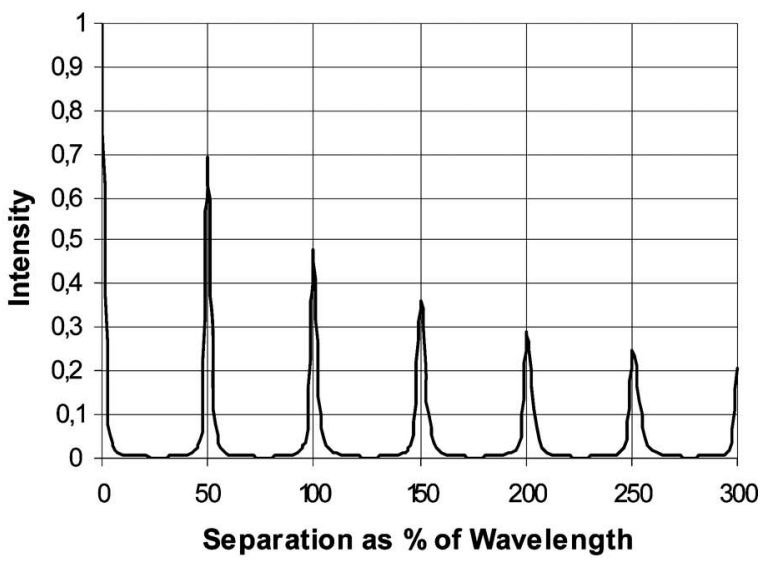

(d)

Fig. 11. Transmission response of the FPI for a variety of dielectric substances and wavelengths. 
gard there has been an evolution toward multimirror FPIs that have two or more coupled cavities with multiple degrees of freedom through the possibility of altering both the reflectivity and the separations of the various components that constitute the FPI. In particular, we look at the three-mirror FPI in two particular configurations based on those found in [5].

In the case of a three-mirror FPI, we find from Eq. (21) that

$$
T=\frac{t_{1}^{2} t_{2}^{2} t_{3}^{2}}{D_{3}}
$$

where the denominator $\mathrm{D}_{3}$ is given by

$$
\begin{aligned}
D_{3}= & 1+\left(r_{1} r_{2}\right)^{2}+\left(r_{2} r_{3}\right)^{2}+\left(r_{1} r_{3}\right)^{2}+2 r_{1} r_{2}\left(1+r_{3}^{2}\right) \cos \left(2 \phi_{1}\right) \\
& +2 r_{2} r_{3}\left(1+r_{1}^{2}\right) \cos \left(2 \phi_{2}\right)+2 r_{1} r_{3} \cos \left(2 \phi_{1}+2 \phi_{2}\right) \\
& +2 r_{1} r_{2}^{2} r_{3} \cos \left(2 \phi_{1}-2 \phi_{2}\right)
\end{aligned}
$$

where the symbols have the usual significance. If one of $r_{1}, r_{2}$, or $r_{3}$ equals 0 , then the two-mirror FPI is produced. Figure 12 illustrates the three-mirror output compared with the equivalent scattering-matrix prediction. As can be seen, there is good agreement between the two cases, indicating that the scattering-matrix technique is reliable when predicting the transmission profile of even complex multicomponent devices. Again the power transmission is calculated by the $S_{21}$ matrix and the reflected power by the $S_{11}$ matrix (see Fig. 1). The frequency of each simulation below is $1 \mathrm{THz}$ to limit the effects of diffraction for ease of comparison to the theory from [5]. The input beam waist width is $W=1 \mathrm{~mm}$ and is located at the surface of the first sheet. All sheets are $\lambda / 4$ and sized to $4 W$ in diameter.

In the case that $r_{1}=r_{3}$ and $\phi_{1}=\phi_{2}$, we find that a flattop, ripple-free transmission with a near-square bandpass, high transmission, and good sideband suppression can be obtained. These are desirable qualities for optical filters and cannot be obtained with a two-mirror FPI. From [5] we find for $r_{1}=r_{3}$ that

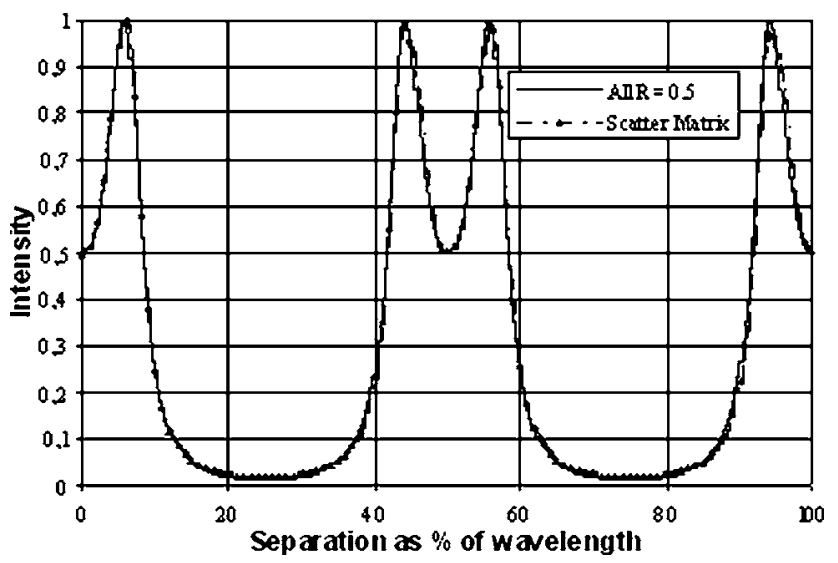

Fig. 12. Transmission profile for a multimirror FPI where all mirrors have the same reflectivity $(R=0.5)$.

$$
T=\frac{\left(1-r_{1}^{2}\right)^{2}\left(1-r_{2}^{2}\right)}{\left(1-r_{1}^{2}\right)^{2}\left(1-r_{2}^{2}\right)+\left[r_{2}\left(1+r_{1}^{2}\right)+2 r_{1} x\right]^{2}}
$$

where $x=\cos (2 \beta)$. When a high value of $r_{2}$ in comparison with $r_{1}$ and $r_{3}$ is chosen, the two cavities are weakly coupled and the double peaks move together, forming a flat-top profile. When a low value is chosen, the two cavities are strongly coupled and the free spectral range will be altered as the fringes separate. This effect is demonstrated in Figs. 13(a) and 13(b), where a choice of $R=0.1$ for the central mirrors and $R=0.5$ for the first and last mirrors separates the peaks and yields four fringes per wavelength, and a choice of $R=0.88$ for the middle mirror pushes the fringes closer and begins to form a flat-top profile. Also shown are the predictions of the scatter-matrix method, which again exhibit excellent agreement throughout the various examples.

A careful choice of $r_{1}, r_{2}$, and $r_{3}$ can yield a near-square bandpass profile. We find from [5] that $r_{1}=r_{3}=0.329$ and $r_{2}=0.739$ are appropriate values for these mirrors. Figure 14 illustrates this profile in comparison with classical theory. Again excellent agreement is found between the two cases with the scatter-matrix technique. The power transmission is effectively the $S_{21}$ matrix, and the reflected power is given in the $S_{11}$ matrix (see Fig. 1).

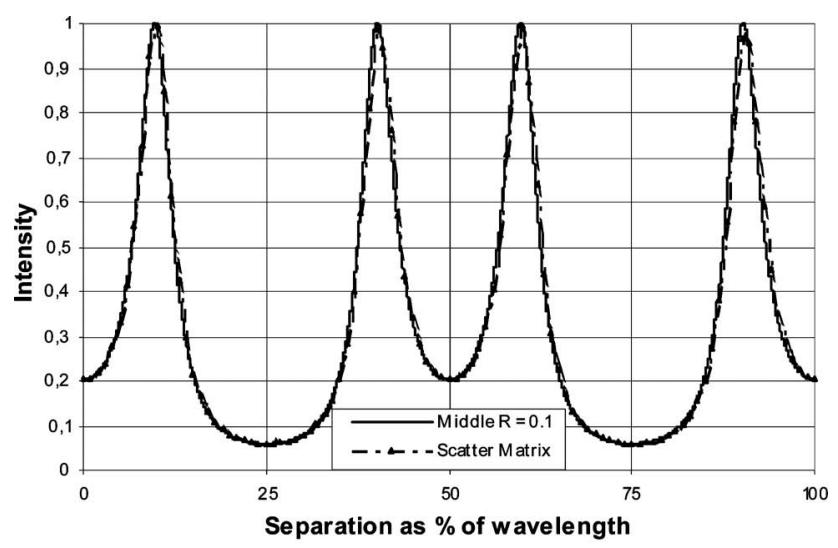

(a)

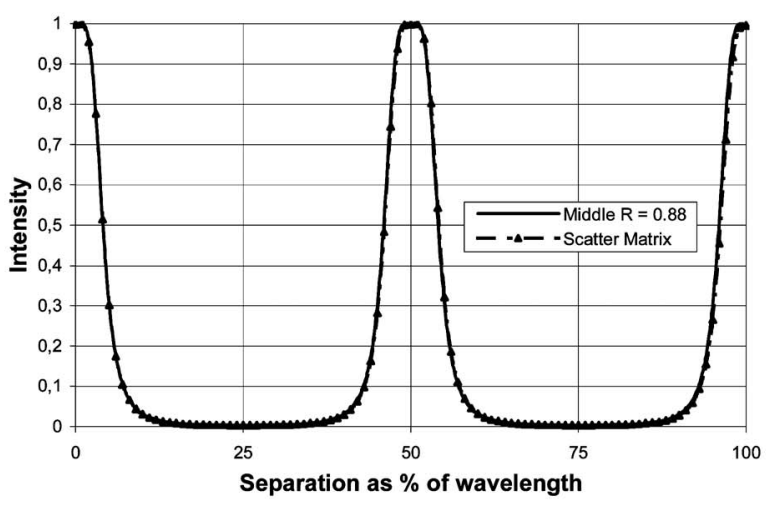

(b)

Fig. 13. (a) Transmission profile for a multimirror FPI where the middle mirror has a reflectivity of $R=0.1$. (b) Transmission profile for a multimirror FPI where the middle mirror has a reflectivity of $R=0.88$. 


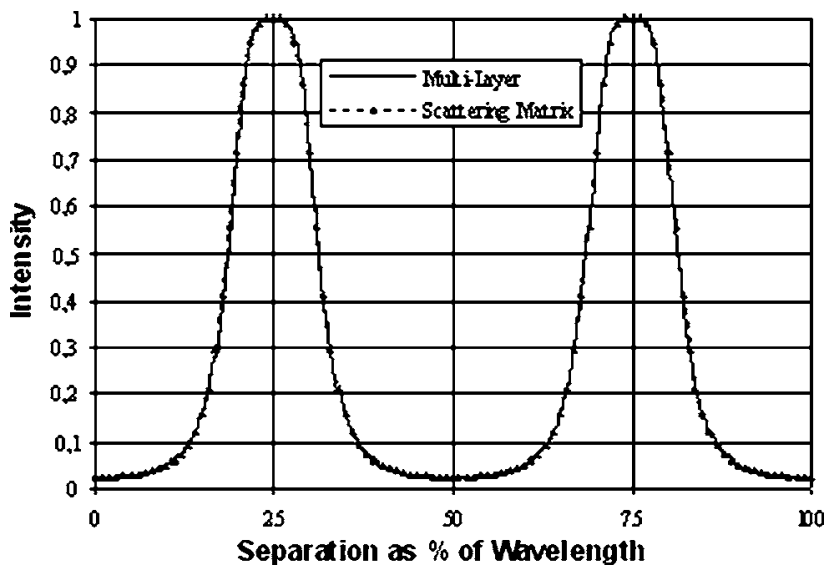

Fig. 14. Response of the multimirror FPI as a bandpass filter with the appropriate choice of reflectivity values.

\section{Fabry-Perot Illumination by Corrugated Horn}

In long-wavelength systems a FPI will be used in conjunction with a quasi-optical feed antenna. As a result, the illumination of the FPI will not be an ideal fundamental Gaussian profile but an actual horn feed beam, which will produce different transmission/reflection characteristics. Here we present an analysis that includes a corrugated horn at $94 \mathrm{GHz}$; the horn is characterized by the following parameters: $W_{\text {aper }}=5.11 \mathrm{~mm}, R_{\text {aper }}=64.8 \mathrm{~mm}$, aperture radius $=2.5 \lambda$, and a flare angle of $5.7^{\circ}$. A full modal description of this antenna was included in the scatteringmatrix framework and subsequently cascaded and transformed to free-space modes. We illustrate a schematic of the experimental setup in Fig. 15.

Theoretical results are compared with experimental measurements, where the horn illuminates two sheets of HDPE. The transmission characteristics of this optical configuration are displayed in Fig. 16. The dielectric sheets that were modeled had a refractive index of 1.52 , which gives a surface reflectivity of $15 \%$, and were $21.5 \mathrm{~mm}$ thick $(6.74 \lambda)$ and $20 \mathrm{~W}$ in diameter. Initially the sheets were in contact with each other as well as with both the source and the detector horn. One horn and dielectric sheet were fixed in place, while the second horn and dielectric sheet were displaced to a distance of $2 \lambda$.

The scatter-matrix technique exhibits excellent agreement with the measured transmission response of the FPI, including the level and size of the ripple. The power coupling has dropped to an average of $6 \%$ as a result of two factors:

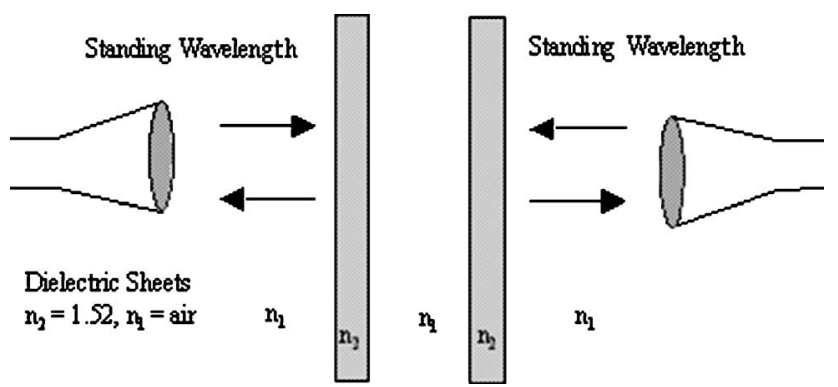

Fig. 15. Experimental setup of two corrugated horns coupled via a FPI.

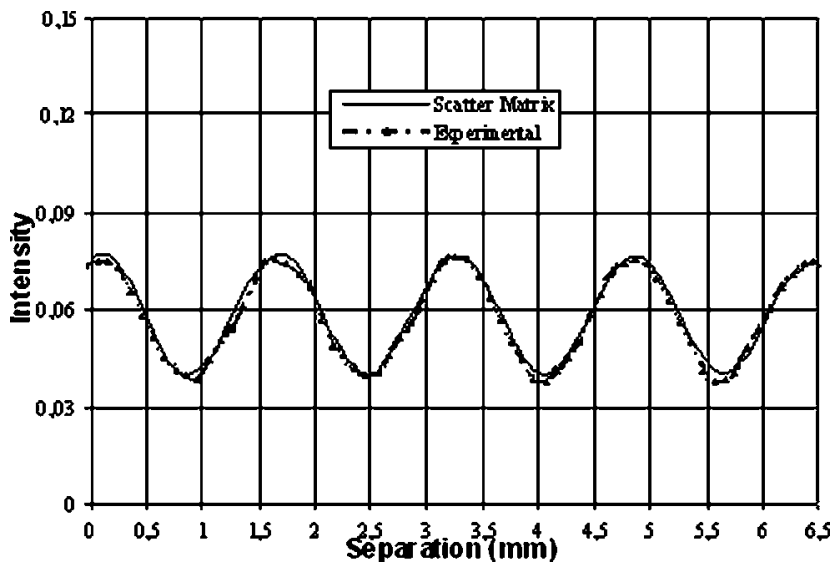

Fig. 16. Power coupling of two corrugated horns coupled via a FPI.

1. The $15 \%$ power loss due to reflected power at each dielectric sheet.

2. The $64 \%$ power loss due to the initial $43 \mathrm{~mm}$ separation of the two corrugated horns.

In Subsections 4.A and 4.B the fundamental Gaussian suffered no losses other than reflective and diffractive losses, as it was not coupling to a detector on the output side of the FPI.

\section{CONCLUSIONS}

In this paper a scattering-matrix formulation based on GBM analysis has been demonstrated to be an accurate predictor of multiple reflections from thin-layer films, including the effects of path length and refractive index variance. This technique can be applied to a wide range of wavelengths, including those considered to be geometrical, in addition to longer wavelengths where diffraction tends to dominate propagation. It has proven to be computationally efficient and can be extended to include the volume of the horn antenna that illuminates the system. The technique incorporates an equivalent waveguide modal description alongside the traditional free-space GBMs in one scattering-matrix formulization. Furthermore, the technique exhibits the ability to predict the transmission characteristics of complex multi-element devices such as the Fabry-Perot interferometer, an advantage over another matrix technique from [5], which cannot include diffraction losses or account for the finite thickness of the dielectric sheets. The real power of the GBM technique stems from the fact that it allows longwavelength behavior to be included while accounting for the structure of the antenna illuminating the systems. A GBM technique for modeling aberrations in off-axis mirror systems that is typically used in a $\mathrm{THz}$ system can be structured as a scattering matrix [12] and is compatible with the GBM scattering-matrix technique presented in this paper. Although clearly this paper has not considered the distortions introduced into the beam by nonideal optics, currently these GBM techniques are being combined, which will potentially allow a full analysis of standingwave and aberration characteristics of optical systems from front-end optics to back-end detectors. 
Although not considered here, the above theory is equally valid for nonnormal-incidence Gaussian beam upon the addition of an appropriate phase term. The device will suffer from lateral walk-off, which will result in less efficient power transfer by the Fabry-Perot interferometer. GBMA is a scalar solution and so neglects polarization effects, whereas a well-designed system should introduce minimal cross-polar effects, which can be estimated [11]. Finally [13,14] are provided to illustrate the importance of GBM in Fabry-Perot laser cavities; these papers discuss the effect of the FPI on the spatial structure of the transmitted beam. Although this topic is not included in this paper, one could reconstruct the beam after propagation through the FPI using the appropriate GBM parameters to evaluate the final beam quality upon transmission.

\section{ACKNOWLEDGMENTS}

Timothy Finn acknowledges a postgraduate research grant awarded by the Irish Research Council for Science, Engineering and Technology (IRCSET) and ESA PRODEX grant funding obtained through Enterprise Ireland.

\section{REFERENCES}

1. J. A. Murphy, N. Trappe, and S. Withington, "Gaussian beam mode analysis of partial reflections in simple quasioptical systems fed by horn antennas," Infrared Phys. Technol. 44, 289-297 (2003).

2. D. Olver, P. Clarricoats, A. Kishka, and L. Shafai,
Microwave Feeds and Horns, Series 39 (IEEE Press, 1994), Chap. 4.

3. E. Gleeson, J. A. Murphy, and B. Maffei, "Phase centers of far infrared multi-moded horn antennas," Int. J. Infrared Millim. Waves 23, 711-730 (2002).

4. N. Trappe, A. Murphy, S. Withington, and W. Jellema, "Gaussian beam mode analysis of standing waves between two coupled corrugated horns," IEEE Trans. Antennas Propag. 53, 1755-1761 (2005).

5. H. Van der Stadt, and J. M. Muller, "Multi-mirror Fabry-Perot Interferometers," J. Opt. Soc. Am. A 2, 1363-1369 (1985).

6. M. Born and E. Wolf, Principles of Optics, 7th ed. (Cambridge U. Press, 1999), Chaps. 1 and 7.

7. D. Martin and J. Bowen, "Long-wave optics," IEEE Trans. Microwave Theory Tech. 41, 1676-1690 (1993).

8. A. Siegman, Lasers (University Science Books, 1986).

9. R. Padman and J. A. Murphy, "A scattering matrix formulation for Gaussian beam-mode analysis," in IEE 7th International Conference on Antennas and Propagation (IEE, 1991), pp. 201-204

10. N. Trappe, T. Finn, A. Murphy, and W. Jellema, "Gaussian beam mode analysis of standing waves in submillimeter telescopes and receiver systems", Proc. SPIE 5497, 137-145 (2004).

11. P. Goldsmith, Quasioptical Systems, Vol. x of IEEE Series on Microwave Techniques and Radio Frequencies (IEEE Press, 1998), Chaps. 3, 5, and 9.

12. J. A. Murphy and S. Withington, "Perturbation analysis of Gaussian beam mode scattering at off-axis ellipsoidal mirrors," Infrared Phys. Technol. 37, 205-219 (1996).

13. F. Moreno and F. González, "Transmission of a Gaussian beam of low divergence through a high-finesse Fabry-Perot device," J. Opt. Soc. Am. A 9, 2173-2175 (1992).

14. H. Y. Abu-Safia, "Transmission of a Gaussian beam through a Fabry-Perot interferometer," Appl. Opt. 33, 3805-3811 (1994). 